\title{
Tightening our understanding of intensive glycaemic control
}

Dr William G. Herrington and Dr David Preiss

Clinical Trial Service Unit and Epidemiological Studies Unit (CTSU), Nuffield Department of Population Health, University of Oxford. will.herrington@ndph.ox.ac.uk

No abstract; Word count: 815 (max. 800); References: 10 (max. 10)

Previous meta-analyses by the Collaborators on Trials of Glucose Lowering (CONTROL) Group have demonstrated the small but potentially important effect of tight glycaemic control on risk of major cardiovascular disease among people with type 2 diabetes within 5 years of intensification of therapy (Table). However, compared to less intensive glycaemic control, this strategy is associated with clear risk of harm from serious hypoglycaemia, which seems to be larger when the $\mathrm{HbA1c}$ target is $6 \%$ compared to $6.5 \%{ }^{1}$

In this month's edition of The Lancet Diabetes and Endocrinology, the CONTROL group now provide confirmation that there are also clearly identifiable benefits on some microvascular complications of diabetes within 5 years. ${ }^{2}$ Using individual participant data, they show a $20 \%$ reduction in markers of kidney disease (a composite of end-stage kidney disease, renal death, sustained eGFR $<30 \mathrm{ml} / \mathrm{min} / 1.73 \mathrm{~m}^{2}$ or sustained macroalbuminuria) and a $13 \%$ reduction in eye complications (a composite of retinal laser therapy or vitrectomy, development of proliferative retinopathy or progression of diabetic retinopathy; Table), but no clear effect on neuropathic complications.

Although somewhat limited by low power, both meta-analyses found no strong statistical evidence that the relative benefits of intensive glycaemic control varied by age or other subgroups. ${ }^{1,2}$ It may therefore be appropriate to sum the benefits on both micro- and macro-vascular disease in the first 5 years of the participating trials and compare them to the potential for severe hypoglycaemia. Such an analysis suggests that overall, tight glycaemic control caused about twice the excess number of severe hypoglycaemic events than the micro- and macro-vascular events it prevented. This reinforces the cautious American Diabetes Association recommendation that "providers might reasonably suggest more stringent $\mathrm{HbA1c}$ goals (such as $<6.5 \%$ ) if this can be achieved without significant hypoglycaemia". ${ }^{3}$ 
It is also important to look beyond the within-trial period as benefits of intensive glycaemic control could continue to emerge after its discontinuation (sometimes referred to as a 'legacy effect'). For microvascular disease, data from the Action to Control Cardiovascular Risk in Diabetes (ACCORD) study suggested accumulation of additional retinopathy benefit in the 4 year post-trial period despite loss of any meaningful difference in \% $\mathrm{HbA1c},{ }^{4}$ albeit based on a subset of 1300 participants.

Emerging post-trial benefits over 3 years on end-stage renal disease have been reported from the Action in Diabetes and Vascular Disease: Preterax and Diamicron MR Controlled Evaluation (ADVANCE) trial in which 8500 participants were followed after the trial. ${ }^{5}$ And, in the United Kingdom Prospective Diabetes Study (UKPDS), the absolute benefits of intensive glycaemic control on microvascular diabetic complications continued to increase after the trial in the comparison of insulin and sulphonylurea therapy to conventional therapy. ${ }^{6}$ However, not all the microvascular outcomes in the trials in CONTROL have demonstrated increasing absolute benefits of intensive glycaemic control beyond the end of the trials. In ADVANCE, no benefit on retinal laser therapy was apparent during or after the trial. ${ }^{5}$

For macrovascular complications, conflicting findings from the post-trial follow-up also exist with one report of a legacy effect, ${ }^{6}$ but other reports not providing confirmation thereof. ${ }^{5,7}$ Therefore, until an individual participant meta-analysis combines all the long-term follow-up data for trials contributing to CONTROL, the full absolute benefits of intensive glycaemic control on micro- and macro-vascular complications, both overall and among subgroups, will remain uncertain. Long-term follow-up of these trials might also allow time for any beneficial effect on 'hard' renal progression endpoints to accrue. More than a half of the primary kidney disease outcomes in the first 5 years of follow-up in the current CONTROL analysis represented development of macroalbuminuria ${ }^{2}$ which, while important, may be considered a surrogate marker of renal progression rather than an outcome of importance to patients.

The latest CONTROL meta-analysis also reveals the substantial risk of microvascular complications which remain despite tight glycaemic control. For diabetic kidney disease, sodium-glucose cotransporter-2 (SGLT-2) inhibitors and human glucagon-like peptide-1 receptor agonists have recently been shown to have potentially important renoprotective effects despite only modest effects on 
glycaemic control, ${ }^{8,9}$ rekindling interest in targeting glomerular hyperfiltration and emphasizing the point that the mechanism may be as important, if not more so, than the extent of glucose lowering achieved. The on-going 4200 patient Evaluation of the Effects of Canagliflozin on Renal and Cardiovascular Outcomes in Participants With Diabetic Nephropathy (CREDENCE) trial is powered to test the effect of SGLT-2 inhibition on a hard cardiorenal primary endpoint (end-stage renal disease, doubling of serum creatinine, renal or cardiovascular death), and the results of this and similar trials will provide important information on how to further reduce micro- and macro-vascular risk in people with diabetes. ${ }^{10}$

In summary, the latest CONTROL meta-analysis has highlighted important but modest microvascular benefits of intensive glucose lowering within 5 years, but extending the meta-analysis to the post-trial period is required for a complete risk:benefit assessment. Moreover, this meta-analysis suggests that research efforts also need to consider other potential pathophysiological mechanisms, as high residual microvascular risk remains despite intensive glycaemic control.

Table: Benefits and harm of more versus less intensive glycaemic control in the 4 large trials contributing to the CONTROL Group meta-analyses within the first 5 years of follow-up

\begin{tabular}{|c|c|c|c|c|c|c|}
\hline & \multicolumn{2}{|c|}{$\begin{array}{l}\text { More intensive } \\
\qquad(n=14,320)\end{array}$} & \multicolumn{2}{|c|}{$\begin{array}{l}\text { Less intensive } \\
\qquad(n=12,729)\end{array}$} & \multirow{2}{*}{$\begin{array}{c}\text { Hazard } \\
\text { ratio }\end{array}$} & \multirow{2}{*}{$\begin{array}{c}95 \% \\
\text { Confidence } \\
\text { interval }\end{array}$} \\
\hline & events & $\%$ & events & $\%$ & & \\
\hline $\begin{array}{l}\text { Known benefit } \\
\text { Major cardiovascular } \\
\text { disease }\end{array}$ & 1194 & $8.3 \%$ & 1176 & $9.2 \%$ & 0.91 & $(0.84-0.99)$ \\
\hline Kidney disease & 761 & $5.3 \%$ & 865 & $6.8 \%$ & 0.80 & $(0.72-0.88)$ \\
\hline Eye disease & 428 & $3.0 \%$ & 367 & $2.9 \%$ & 0.87 & $(0.76-1.00)$ \\
\hline \multicolumn{7}{|l|}{ Harm } \\
\hline Severe hypoglycaemia & 1071 & $7.5 \%$ & 372 & $2.9 \%$ & 2.48 & $(1.91-3.21)$ \\
\hline Any death & 980 & $6.8 \%$ & 884 & $6.9 \%$ & 1.04 & $(0.90-1.20)$ \\
\hline
\end{tabular}
Adapted from CONTROL Group meta-analyses ${ }^{1,2}$ 


\section{Conflicts of interest}

The author declared no conflicts of interest

\section{References}

1. CONTROL Group. Intensive glucose control and macrovascular outcomes in type 2 diabetes. Diabetologia 2009; 52(11): 2288-98.

2. CONTROL Group. The effects of intensive glucose control on microvascular outcomes in patients with type 2 diabetes: a meta-analysis of individual participant data from randomised controlled trials. The Lancet Diabetes \& Endocrinology 2017.

3. American Diabetes Association. Glycemic targets. Diabetes Care 2015; 38(supppl. 1): S33S40.

4. Action to Control Cardiovascular Risk in Diabetes Follow-On Eye Study Group. Persistent Effects of Intensive Glycemic Control on Retinopathy in Type 2 Diabetes in the Action to Control Cardiovascular Risk in Diabetes (ACCORD) Follow-On Study. Diabetes Care 2016; 39(7): 1089-100. 5. Zoungas S, Chalmers J, Neal B, et al. Follow-up of blood-pressure lowering and glucose control in type 2 diabetes. N Engl J Med 2014; 371(15): 1392-406.

6. Holman RR, Paul SK, Bethel MA, Matthews DR, Neil HA. 10-year follow-up of intensive glucose control in type 2 diabetes. N Engl J Med 2008; 359(15): 1577-89.

7. Hayward RA, Reaven PD, Emanuele NV et al. Follow-up of Glycemic Control and Cardiovascular Outcomes in Type 2 Diabetes. N Engl J Med 2015; 373(10): 978.

8. Wanner C, Inzucchi SE, Lachin JM, et al. Empagliflozin and Progression of Kidney Disease in Type 2 Diabetes. N Engl J Med 2016.

9. Marso SP, Daniels GH, Brown-Frandsen K, et al. Liraglutide and Cardiovascular Outcomes in Type 2 Diabetes. N Engl J Med 2016; 375(4): 311-22.

10. Evaluation of the Effects of Canagliflozin on Renal and Cardiovascular Outcomes in Participants With Diabetic Nephropathy (CREDENCE). https://clinicaltrials.gov/ct2/show/NCT02065791 (accessed 10th February 2017). 